\title{
Four-year follow-up outcomes after stereotactic body radiation therapy for central early-stage non-small cell lung cancer
}

\author{
Xiaojiang Sun ${ }^{1 \#}$, Yefei $\mathrm{Li}^{2 \#}$, Yaoyao Zhu ${ }^{2,3 \#, ~ Q i a n ~} \mathrm{Li}^{2}$, Xiaoshuai Yuan ${ }^{2}$, Qingren Lin ${ }^{1}$, Denghu Weng ${ }^{1}$, \\ Qinghua $\mathrm{Xu}^{2}$, Hui Liu ${ }^{2}$, Yaping $\mathrm{Xu}^{1,2,3}$ \\ ${ }^{1}$ Department of Radiation Oncology, Cancer Hospital of University of Chinese Academy of Sciences (Zhejiang Cancer Hospital), Institute of Cancer \\ and Basic Medicine (IBMC), Chinese Academy of Sciences, Hangzhou, China; ${ }^{2}$ Department of Radiation Oncology, Shanghai Pulmonary Hospital, \\ Tongji University School of Medicine, Shanghai, China; ${ }^{3}$ First Clinical Medical School, Wenzhou Medical University, Wenzhou, China \\ Contributions: (I) Conception and design: Y Xu, H Liu; (II) Administrative support: Y Xu, X Sun; (III) Provision of study materials or patients: X Sun, \\ Q Xu, Q Lin; (IV) Collection and assembly of data: X Sun, Q Li, X Yuan, D Weng, Q Xu; (V) Data analysis and interpretation: X Sun, Y Li, Y Zhu; (VI) \\ Manuscript writing: All authors; (VII) Final approval of manuscript: All authors. \\ \#These authors contributed equally to this work. \\ Correspondence to: Yaping Xu; Hui Liu; Qinghua Xu. Department of Radiation Oncology, Shanghai Pulmonary Hospital, Tongji University School of \\ Medicine; No. 507 Zhengmin Road, Shanghai, China. Email: xuyaping1207@163.com; huiliu1127@163.com; qinghuaxu@126.com.
}

Background: Previous research has shown that stereotactic body radiation therapy (SBRT) can achieve a high level of tumor control in patients with early-stage non-small cell lung cancer (NSCLC). However, to date, such studies have mainly focused on peripheral early-stage patients. This study aimed to assess the clinical outcomes and toxicity of patients with central lung cancer treated with SBRT in our institution.

Methods: A total of 31 consecutive central early-stage NSCLC patients who were treated with SBRT using the biologically effective dose (BED; $\alpha / \beta=10)$ 100-119 Gy in 4-10 fractions between April, 2013, and August, 2016, were reviewed. The RTOG 0813 trial standard was used to define whether the NSCLC was centrally located. All patients received four-dimensional computed tomography (4D CT) simulation. Intensity modulated radiation therapy (IMRT) and three-dimensional conformal radiation therapy (3D CRT) techniques were used in treatment planning. The dose to the planning target volume (PTV) was prescribed to the $95 \%$ isodose line. Mainly dosimetric parameters, clinical outcomes, and toxicity were analyzed.

Results: The 31 patients enrolled in the study had a median follow-up time of 47.7 months, and the median tumor diameter was $2.2 \mathrm{~cm}$ (range, $1.3-5.0 \mathrm{~cm}$ ). A total of 15 patients $(48.4 \%$ ) developed disease recurrence. The incidences of local, regional, and distant disease recurrence at 3 years were $11.7 \%, 9.7 \%$, and $30.7 \%$, respectively; at 5 years, they were $21.5 \%, 15.0 \%$, and $35.0 \%$, respectively. The 3 - and 5 -year progression-free survival (PFS) rates were $55.1 \%$ and $40.5 \%$, respectively; the corresponding overall survival (OS) rates were $85.3 \%$ and $68.4 \%$, respectively. Toxicities of grade 3 or higher were observed in $6.5 \%$ of the patients. None of the patients experienced grade 4 or 5 acute adverse events; however, 2 patients possibly died of treatment-related late toxicity.

Conclusions: SBRT with a BED 100 Gy in 4-10 fractions is effective and acceptable for treating patients with central early-stage NSCLC. Further studies are warranted.

Keywords: Non-small cell lung cancer (NSCLC); outcome; stereotactic body radiotherapy; toxicity; central tumor

Submitted Apr 26, 2020. Accepted for publication Aug 06, 2020.

doi: $10.21037 /$ tlcr-20-851

View this article at: http://dx.doi.org/10.21037/tlcr-20-851 


\section{Introduction}

Lung cancer kills more people than any other cancer worldwide. Non-small cell lung cancer (NSCLC) comprises approximately $85 \%$ of all lung cancer cases (1). Surgery is the first choice of treatment for operable earlystage lung cancer. Stereotactic body radiation therapy (SBRT), as a high-precision, low-toxic radiation therapy, has achieved great progress in early-stage NSCLC. Compared with conventional standard radiotherapy, SBRT can achieve a high level of local control (LC) of the primary disease without increasing toxicity in short treatment times (2). For SBRT, compared with less intensive regimens, intensive regimens of $\mathrm{BED} \geq 100$ Gy are related with remarkably better local control and survival (3). SBRT is recommended as a treatment of choice for patients with medically inoperable early-stage peripheral lung cancer and is most commonly used for tumors up to $5 \mathrm{~cm}$ in size. However, controversy still surrounds the outcomes and safety of SBRT for patients with central lung cancer.

Treating central tumors (within $2 \mathrm{~cm}$ of the carina) is challenging. Because of toxicity, many studies have focused on the treatment of central tumors. In Timmerman's phase II study, the total SBRT treatment dose was 60-66 Gy, delivered in 3 fractions over 1-2 weeks; 6 of 70 patients died as a result of treatment-related toxicity (4). The landmark RTOG 0236 trial did not include patients with cancer located in the central lung (5), which at that time was referred to as the "no fly zone". More recently, the RTOG 0813 reported that it is safe and effective to use SBRT in central NSCLC. In that study, patients were treated with a modified SBRT regimen with 50-60 Gy divided into 5 fractions. In RTOG 0813, the definition of central lung cancer was expanded to include tumors within or around the proximal bronchial tree (PBT), including the trachea, the pericardium, mediastinum, and the spine, and the results indicated good efficacy and safety despite the occurrence of grade $\geq 3$ toxicity (6).

Since the optimal dose regimen for the treatment of central tumors is unclear, various regimens have been used around the world based on institutional experience. Published research has reported diverse regimes using biologically effective doses (BEDs) of 43-180 Gy (6-11). Moreover, the experience of using SBRT in clinical practice in China is particularly limited. Herein, taking this uncertainty into consideration, we report our institutional experience of SBRT for central tumors.
We present the following article in accordance with the STROBE reporting checklist (available at http://dx.doi. org/10.21037/tlcr-20-851).

\section{Methods}

\section{Patient characteristics}

The data of 31 consecutive central early-stage NSCLC patients who received SBRT in our hospital between April, 2013, and August, 2016, were retrospectively analyzed. Central tumors were defined as those within or touching the area of the PBT (within $2 \mathrm{~cm}$ of the PBT or immediately adjacent to the mediastinal/pericardial pleura). The included patients all had an Eastern Cooperative Oncology Group (ECOG) performance status score of between 0 and 2 , and stage T1-2N0M0 tumors according to the seventh edition of American Joint Committee on Cancer (AJCC) staging system. Each patient underwent computed tomography (CT) and positron emission tomographycomputed tomography (PET-CT) scans in the four weeks before registration. All of the patients were assessed as medically inoperable by an experienced thoracic surgeon. Patients whose tumors had previously not been irradiated were included, as were those with cytologic or histologic proof of primary NSCLC or with a clinical diagnosis according to medical history and imaging examinations. There were no restrictions on pulmonary function tests. The study protocol was approved by the human research ethics committee of our institution, and signed informed consent was obtained from all patients. The study was conducted in accordance with the Declaration of Helsinki (as revised in 2013).

\section{SBRT treatment}

All patients underwent four-dimensional CT (4D CT)based simulation. If the tumor moved more than $1.5 \mathrm{~cm}$, respiratory gating was considered. The patients were immobilized using a vacuum bag. At our center, the gross tumor volume (GTV) is defined as the pulmonary tumor observed on CT imaging by maximal intensity projection. The internal target volume (ITV) was contoured based on the GTV and edited at the lung window based on 4D-CT to include the full movement of the tumor. The planned target volume (PTV) was defined as the ITV plus a $5-\mathrm{mm}$ setup margin and was modified at the discretion of the physician. The dose prescription was chosen to ensure $95 \%$, 
$99 \%$, and $100 \%$ of the PTV, ITV, respectively, received the prescribed dose. Organs at risk and dosimetric limits included the spinal cord, esophagus, heart, normal lung, trachea, and the PBT. The dosimetric parameters were calculated using the treatment planning system (RayStation version 4.5.1, RaySearch Laboratories, Stockholm, Sweden). Treatment was delivered using a $6-M V$ or $10-M V$ linear accelerator with either multiple static intensity modulated radiation therapy or three-dimensional (3D) conformal treatment. Cone beam CT was implemented before each treatment fraction. According to the dose prescribed by the treating and chief physicians, medical physicists formulated treatment plans for each patient. According to the experience of Timmerman et al. in 2011, dose constraints were implemented to critical structures (12).

\section{Follow-up and outcome assessment}

All patients were advised to attend regular follow-up examinations at our hospital. The first follow-up was performed one month after SBRT, then every three months for two years, and every six months thereafter. Examinations included routine blood tests, B-ultrasonography of the supraclavicular lymph nodes and abdomen, and contrastenhanced CT of the chest. PET-CT scans were performed when it was not clear whether the disease was progressing. Patients who attended follow-up at local hospitals were contacted and assessed by the treating physicians. The infield effect was evaluated according to the Response Evaluation Criteria in Solid Tumors (RECIST), version 1.1, while treatment-related toxicity was scored according to the Common Terminology Criteria for Adverse Events (CTCAE), version 5.0. An adverse event occurring $<3$ months after radiotherapy was defined as acute toxicity, and an event occurring $>3$ months after radiotherapy was defined as late toxicity. When a single patient had multiple reports of toxicity, only the highest score was included in the analysis. Different radiation oncologists reviewed deaths with an unknown cause that were possibly related to treatment and scored as a grade 5 toxicity if they were found to be related to treatment. The follow-up duration was measured from the first day of SBRT to death or the date of last follow-up. Events of interest included LC, regional progression-free time (RPFT) and distant progression-free time (DPFT), progression-free survival (PFS), and overall survival (OS), which were related to local failure, regional lymph node metastasis, distant failure, disease progression or death, and death, respectively. The LC, RPFT, DPFT, PFS, OS, and toxicity rates were calculated from the starting day of SBRT until the occurrence of an event of interest, or death or last follow-up.

\section{Statistical methods}

Descriptive statistics were expressed as median values, frequency, and percentages. The Kaplan-Meier method was employed to generate survival curves for LC, RPFT, DPFT, PFS, and OS. The potential predictive factors of PFS and OS were identified with univariate and multivariate Cox proportional hazard regression models. A $\mathrm{P}$ value of $<0.05$ was considered statistically significant. Statistical analyses were performed with SPSS software (version 22.0, SPSS Inc., Chicago, Illinois, USA).

\section{Results}

\section{Patient and tumor characteristics}

A total of 31 central early-stage NSCLC patients were enrolled in this study. The tumors seen on enhanced lung CT and fluorine-18-fluorodeoxyglucose $\left({ }^{18} \mathrm{~F}-\mathrm{FDG}\right) \mathrm{PET} /$ CT were treated with SBRT. Of the 24 patients whose lesions were touching the $2-\mathrm{cm}$ area around the PBT, 19 patients had lesions adjacent to the mediastinal pleura, 5 patients had lesions adjacent to the pericardial pleura, and 13 patients' lesions were ultra-central. The median age of the patients was 75 years (range, $57-88$ years). NSCLC was pathologically proven in 22 patients $(71.0 \%)$, and 6 patients $(19.4 \%)$ had squamous-cell carcinoma. The median diameter of the tumors was $2.2 \mathrm{~cm}$ (range, $1.3-5.0 \mathrm{~cm}$ ). Table 1 summarizes the pretreatment characteristics and outcomes of the patients.

\section{Radiotherapy treatment details}

Table 2 lists the characteristics of the radiotherapy treatment received by the patients. Representative CT images of SBRT for central tumors are shown in Figure S1. The median dose of SBRT was $50 \mathrm{~Gy} / 5$ fractions and the median BED $(\alpha / \beta=10)$ was 100 Gy (range, $100-119$ Gy). The median volume of the PTV was $35.0 \mathrm{~cm}^{3}$ (range, 10.5 $\left.122.0 \mathrm{~cm}^{3}\right)$.

\section{Clinical outcomes}

Figure $1 A, B$ show the Kaplan-Meier curves of LR, RR, 
Table 1 The pretreatment characteristics and outcomes of the 31 enrolled early-stage NSCLC patients

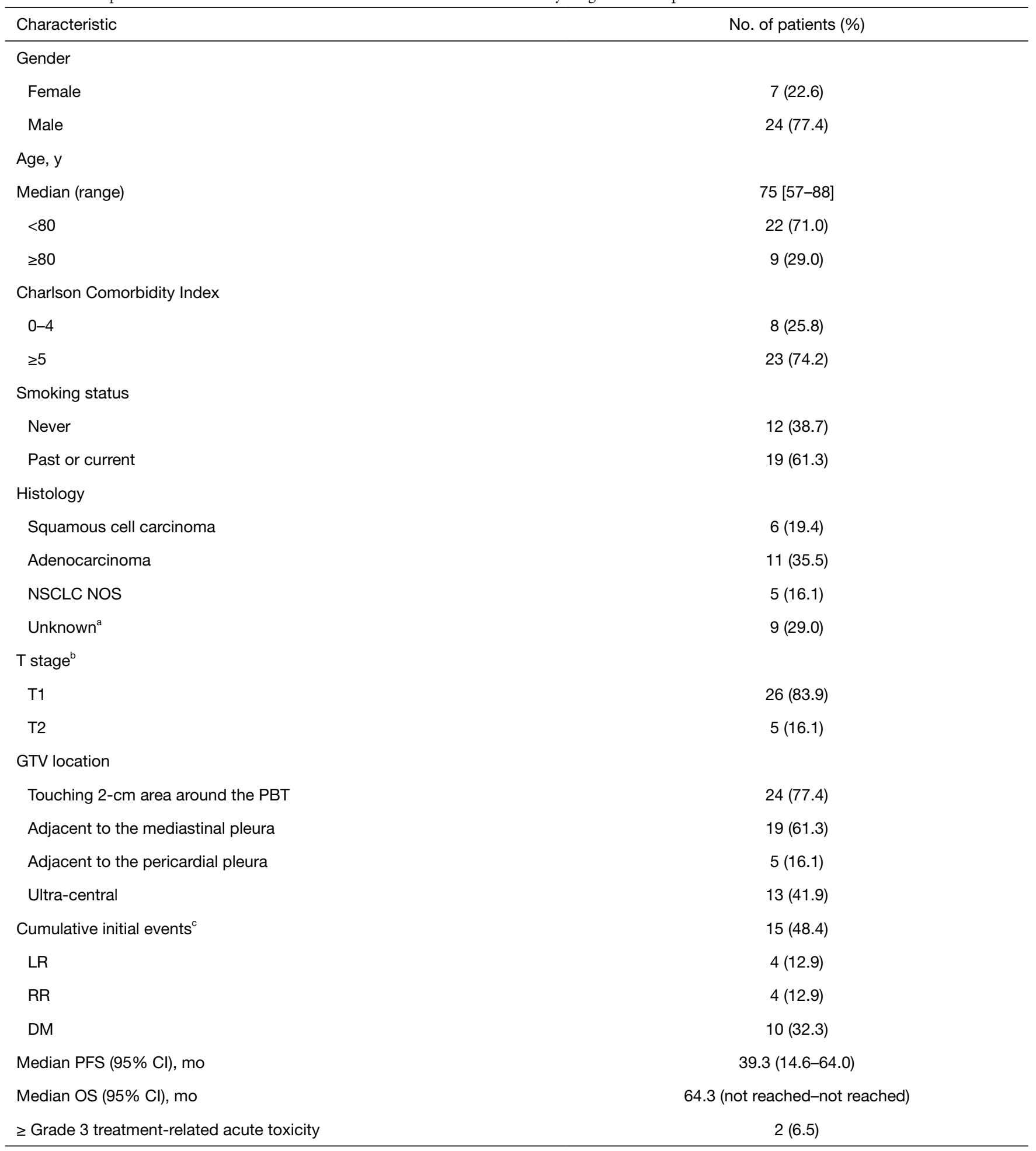

\footnotetext{
${ }^{\mathrm{a}}$, patients were clinically diagnosed according to their medical history and imaging examinations after refusing a biopsy; ${ }^{b}$, tumors no larger than $5 \mathrm{~cm}$; ${ }^{\text {, }}$, three patients suffered simultaneous failure (one had RR and DM; one had all-sites failure). NSCLC, non-small cell lung cancer; NOS, not otherwise specified; GTV, gross tumor volume; PBT, proximal bronchial tree; LR, local disease recurrence; RR, regional disease recurrence; DM, distant metastasis; PFS, progression-free survival; OS, overall survival.
} 
Table 2 Treatment characteristics

\begin{tabular}{lc}
\hline Characteristic & No. of patients (\%) \\
\hline Fractionation & $1(3.2)$ \\
70 Gy/7 Gy fx. & $1(3.2)$ \\
60 Gy/7.5 Gy fx. & $25(80.6)$ \\
50 Gy/10 Gy fx. & $1(3.2)$ \\
55 Gy/11 Gy fx. & $3(9.7)$ \\
50 Gy/12.5 Gy fx. & \\
PTV & $35.0(10.5-122.0)$ \\
PTV (cc), median (range) & \\
Lung dose parameters & $3.51(1.0-6.6)$ \\
Mean lung dose (Gy), median (range) & $16.3(5.3-26.9)$ \\
V5 (\%), median (range) & $10.1(3.2-20.1)$ \\
V10 (\%), median (range) & $4.5(1.7-10.2)$ \\
V20 (\%), median (range) & $277.3(74.8-537.0)$ \\
V12.5 (cc), median (range) & $251.0(70.1-488.0)$ \\
V13.5 (cc), median (range)
\end{tabular}

DM, PFS, and OS. At last follow-up (February 20, 2020), 8 patients $(25.8 \%)$ had died. The median follow-up interval was 47.7 months (range, 6.5-79.6 months).

Disease progression occurred in $48.4 \%$ (15/31) of patients, and 4 (12.9\%), 4 (12.9\%), and $10(32.3 \%)$ patients experienced local, regional, and distant failure, respectively. Among these patients, one patient had local recurrence and regional metastasis, and one had local and regional recurrence as well as distant metastasis. Of the 15 patients whose disease progressed, 8 patients were treated: 3 patients received systemic therapy including chemotherapy and targeted therapy; 1 patient received chemotherapy, radioactive seed implantation, and radiofrequency ablation; 1 patient received targeted therapy and palliative radiotherapy for bone metastases; 1 patient received chemotherapy and lung re-irradiation with SBRT; 1 patient received palliative radiotherapy for brain metastases; and 1 patient received mediastinal lymph node radiotherapy. At 3 years, the estimated cumulative incidences of local, regional, and distant disease recurrence were $11.7 \%$, $9.7 \%$, and $30.7 \%$, respectively; and at 5 years they were

B

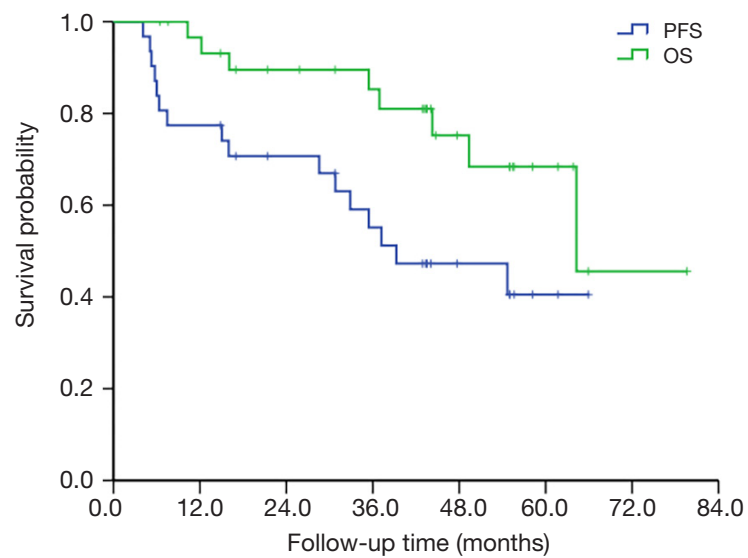

No. at risk

$\begin{array}{lllllllll}\text { PFS event } & 31 & 24 & 19 & 14 & 7 & 2 & 0 & 0\end{array}$

$\begin{array}{lllllllll}\text { Death } & 31 & 28 & 23 & 20 & 11 & 5 & 1 & 1\end{array}$

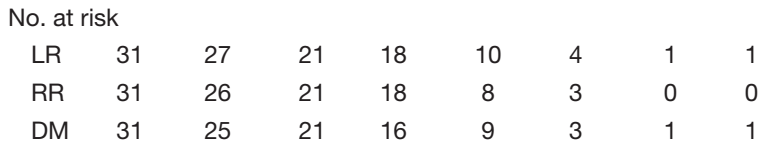

Figure 1 Outcomes of stereotactic body radiation therapy for central non-small cell lung cancer. Kaplan-Meier curves for early-stage patients: (A) local disease recurrence (LR), regional disease recurrence (RR) and distant metastasis (DM); (B) progression-free survival (PFS) and overall survival (OS). 
Table 3 Clinicopathological characteristics of the 8 patients who died

\begin{tabular}{cccccccccccc}
\hline No. Gender Age, y & $\begin{array}{c}\text { ECOG } \\
\text { PS }\end{array}$ & CCl & $\begin{array}{c}\text { Smoking } \\
\text { status }\end{array}$ & $\begin{array}{c}\text { Lung disease } \\
\text { before SBRT }\end{array}$ & Histology & Size, cm & Dosage ${ }^{a}$ & Survival, mo & Cause of death \\
\hline 1 & M & 78 & 1 & 5 & 1 & No & NSCLC NOS & 2.20 & $12.5 \times 4$ & 44.3 & Lung cancer \\
2 & W & 73 & 2 & 6 & 0 & Yes & AC & 1.50 & $10 \times 5$ & 35.4 & Cerebral infarction \\
3 & W & 67 & 1 & 4 & 0 & No & AC & 1.40 & $10 \times 5$ & 64.3 & Cerebral hemorrhage \\
4 & M & 82 & 2 & 6 & 1 & Yes & SCC & 5.00 & $10 \times 5$ & 49.4 & Lung cancer \\
5 & M & 60 & 1 & 5 & 1 & Yes & AC & 1.90 & $10 \times 5$ & 10.3 & Lung cancer \\
6 & M & 80 & 0 & 5 & 0 & No & AC & 2.20 & $10 \times 5$ & 36.9 & Myocardial infarction \\
7 & M & 79 & 0 & 5 & 1 & No & AC & 2.00 & $10 \times 5$ & 12.2 & Treatment-related cau \\
8 & M & 82 & 1 & 6 & 1 & Yes & SCC & 2.80 & $10 \times 5$ & 16.1 & Treatment-related cau \\
\hline
\end{tabular}

a, dose in Gy x number of fractions. ECOG, Eastern Cooperative Oncology Group; PS, performance status; CCI, Charlson Comorbidity Index; NSCLC, non-small cell lung cancer; NOS, not otherwise specified; M, man; W, woman; AC, adenocarcinoma; SCC, squamous cell carcinoma; cau, cause.

$21.5 \%, 15.0 \%$, and $35.0 \%$, respectively. The median PFS for the patients in this study was 39.3 months [95\% confidence interval (CI), 14.7-63.9 months]. The 3- and 5 -year PFS rates were $55.1 \%$ and $40.5 \%$, respectively. Eight patients (25.8\%) died, of whom 3 were considered to have died from cancer-related causes, including distant metastases to the lung, liver, or brain. Two patients who died at home of unknown causes were considered to have possibly developed respiratory failure, which was scored as a grade 5 late toxicity after the patients' medical histories were reviewed by two radiation oncologists during followup. Three patients died of other diseases, including cerebral infarction (one patient), cerebral hemorrhage (one patient), and myocardial infarction (one patient). Table 3 displays the clinicopathological characteristics of the 8 patients who died during follow-up. The median OS was 64.3 months (95\% CI, not reached-not reached). The 3and 5 -year OS rates were $85.3 \%$, and $68.4 \%$, respectively. In the multivariate analysis, smoking $(\mathrm{P}=0.041)$, Charlson Comorbidity Index $\geq 5 \quad(\mathrm{P}=0.046)$, and adenocarcinoma $(\mathrm{P}=0.035)$ were significantly associated with shorter OS. Smoking $(\mathrm{P}=0.027)$ and Charlson Comorbidity Index $\geq 5$ $(\mathrm{P}=0.032)$ was significantly associated with shorter PFS. The Cox proportional hazard regression model was used in a multivariate analysis of PFS and OS (Table 4).

\section{Toxicity}

Two (6.5\%) of the 31 patients experienced grade $\geq 3$ acute toxicity. Both of these patients suffered grade 3 radiation pneumonitis. No acute grade 4-5 toxicity was reported. Two patients suffered grade 5 late toxicity.

\section{Discussion}

The use of SBRT in the treatment of early-stage inoperable NSCLC can achieve excellent LC and has tolerable levels of toxicity. However, these results have mainly been observed in patients with peripheral tumors. In the treatment of central tumors with SBRT, optimizing the risk-benefit ratio is still an area that is being actively explored. This is because the risk of toxicity with SBRT for central tumors is higher than that for peripheral tumors. To date, the long-time profile of the efficacy and safety of SBRT for central earlystage NSCLC has not been well concluded. Moreover, the experience of using SBRT in clinical practice in China is particularly limited.

In this retrospective 4-year study, we investigated the efficacy of SBRT BED10 100-119 Gy delivered in 4-10 fractions for patients with central early-stage NSCLC with a 4-year median follow-up. To our knowledge, the current study has the longest follow-up time of the studies exploring the use of SBRT in central early-stage NSCLC patients. We observed outstanding outcomes, with low incidences of $\mathrm{LR}, \mathrm{RR}$, and $\mathrm{DM}$ (21.4\%, $15.0 \%$ and $35.0 \%$, respectively), and encouraging 5 -year PFS and OS rates ( $40.5 \%$ and $68.4 \%$, respectively). The incidence of grade $\geq 3$ acute adverse events was extremely low; only 2 patients 
Table 4 Multivariate cox proportional hazard regression analysis of PFS and OS for the clinicopathological features of the 31 patients

\begin{tabular}{|c|c|c|c|c|}
\hline Clinical factors & \multicolumn{2}{|c|}{ PFS } & \multicolumn{2}{|l|}{ OS } \\
\hline \multicolumn{5}{|l|}{ Gender } \\
\hline Female & 1.00 & & 1.00 & \\
\hline Male & $0.52(0.09-2.87)$ & 0.449 & $0.93(0.04-24.66)$ & 0.964 \\
\hline$<80$ & 1.00 & & 1.00 & \\
\hline$\geq 80$ & $0.89(0.22-3.66)$ & 0.868 & $2.14(0.20-23.48)$ & 0.534 \\
\hline \multicolumn{5}{|l|}{ Smoking status } \\
\hline Never & 1.00 & & 1.00 & \\
\hline $0-4$ & 1.00 & & 1.00 & \\
\hline$\geq 5$ & $6.15(1.17-32.32)$ & 0.032 & $13.09(1.05-175.87)$ & 0.046 \\
\hline \multicolumn{5}{|l|}{ Histology } \\
\hline Squamous carcinoma & 1.00 & & 1.00 & \\
\hline Adenocarcinoma & $2.81(0.51-15.31)$ & 0.233 & $34.28(1.28-918.99)$ & 0.035 \\
\hline Others & $0.36(0.09-1.54)$ & 0.169 & $0.26(0.02-2.91)$ & 0.272 \\
\hline
\end{tabular}

$\mathrm{HR}$, hazard ratio; Cl, confidence interval; PFS, progression-free survival; OS, overall survival.

experienced grade 3 acute radiation pneumonitis (6.5\%), and no grade 4 or 5 acute adverse events occurred.

The outcomes of recently published reports for central lung cancer are listed in Table 5 (6-11). Most of these studies reported that SBRT achieved good LC and OS rates in central NSCLC; however, Schanne et al. reported 3-year LC and OS rates of only $52 \%$ and $29 \%$, respectively (11). This difference may be explained by the wide range of doses in these studies; the median BED10 of 72 Gy given by Schanne was lower than those in most other studies (median $\mathrm{BED} \geq 100 \mathrm{~Gy})$. The LC rate in the current study was higher than in the previous studies, which may be attributable to BED10 $\geq 100$ Gy carrying a longer LC and a lower risk of recurrence and metastasis compared to BED10 <100 Gy. Furthermore, the PTV in our study was relatively small, with a median volume of $35.0 \mathrm{~cm}^{3}$ (range, $10.5-122.0 \mathrm{~cm}^{3}$ ), which could have also contributed to the lower rate of tumor recurrence. It has been reported that a higher radiation dose delivered to the PTV and a smaller tumor are related with better outcomes $(13,14)$. However, the initial disease failure pattern indicated that DM is still the predominant type of failure, which suggests that attention should be paid to systemic treatment in central earlystage NSCLC patients. More importantly, treatments that effectively eradicate occult metastases would change the disease status of patients and improve their prognosis. The use of concurrent or adjuvant chemotherapy in NSCLC patients treated with SBRT remains to be explored. In a cohort of T1-3N0M0 NSCLC patients who were treated with SBRT (20 patients with T1, 34 with T2, and 11 with T3), Chen et al. (15) treated 17 patients with cisplatincontaining adjuvant chemotherapy after SBRT. They found that chemotherapy after SBRT decreased the risk of tumor recurrence by $29 \%$ and increased the 5 -year OS rate by $14 \%$, without causing any severe infections or other toxicities. Recently, a combination of an immunotherapy and SBRT has been proposed based on evidence that immunotherapy changes the metastatic environment and may be better for eliminating micrometastasis (16-18). The PACIFIC 4 study, a phase II prospective randomized trial to compare SBRT alone with SBRT combined immunotherapy, is currently ongoing. This much needed 


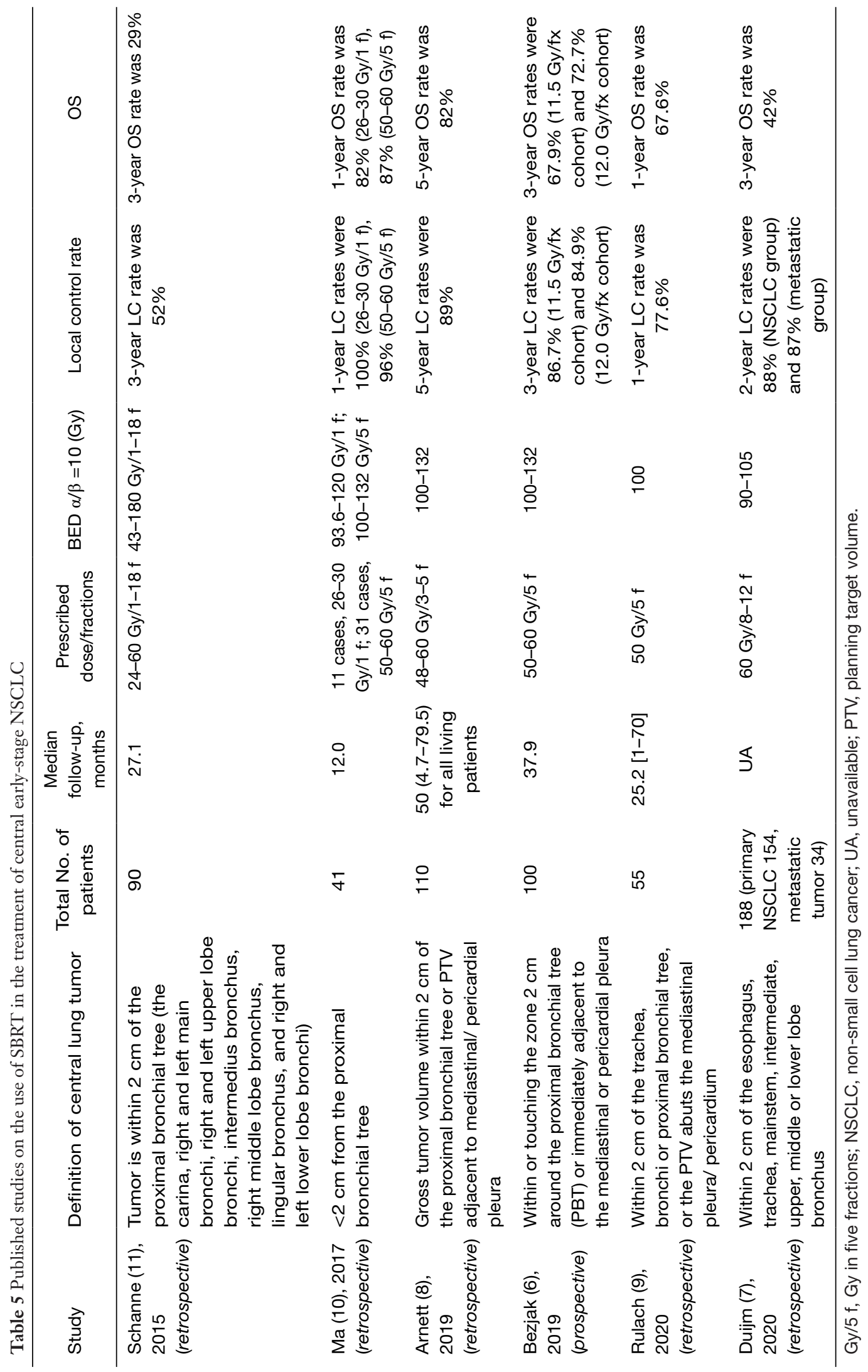


study may help to emphasize the importance of treating visible and occult lesions, and further enhance the prognosis of early-stage NSCLC patients who receive SBRT (19).

No grade 5 toxicities that were reported with SBRT in other studies on central tumors, including fatal haemoptysis, bronchial strictures and fistulae, and hemorrhage esophageal perforation, were observed in current study. Tumors that are more centrally located could possibly lead to a greater dose to the aforementioned critical structures, which may result in a higher risk of toxicity. The Nordic HILUS trial, the results of which have yet to be fully reported, treated patients with tumors $<1 \mathrm{~cm}$ from the PBT with $56 \mathrm{~Gy} / 8$ fractions. There is currently no survival data available from the study, but the incidence of grade 3-5 toxicity was $28 \%$, including $9.5 \%$ of all patients who experienced fatal events (primarily hemoptysis) (20). The majority of tumors irradiated in our study were located $\geq 1.0 \mathrm{~cm}$ away from the PBT, which may account for the low incidence of toxicity observed in our cohort. Cases with tumors located closer to the PBT are likely to see a greater dose to the fatal structures, resulting in a higher risk of toxicity. In addition, a moderate BED dose scheme (BED10 100-119 Gy) was applied in our study. It has been reported in RTOG 0813 that a moderate radiation dose delivered (10-11.5 Gy/f, $5 \mathrm{f}$ ) to the PTV is associated with a lower risk of toxicity compared with higher radiation dose (6). Notably, the possibility for high-grade late toxicity should not be overlooked. Our study reported two deaths from respiratory failure that were possibly related to treatment, which occurred at 16.1 and 12.2 months after SBRT. Despite the difficulty in determining whether the deaths of these two patients were caused by SBRT toxicity, it is possible for high-risk patients who receive SBRT to encounter severe toxicity $>12$ months after treatment. Recently, Duijm et al. who treated 188 central lung tumors with SBRT (60 Gy/8 fractions or $60 \mathrm{~Gy} / 12$ fractions), reported that 4 patients suffered late severe toxicity, including 2 deaths related to the treatment (1 patients died of a tracheoesophageal fistula 10 months after irradiation, and the other patient's death was attributed to septic mediastinitis caused by a large esophagus perforation at 14 months after SBRT), while no acute severe esophageal toxicity was reported (7). Therefore, a moderate dose of BED10 100 might not cause severe acute toxicity. Caution should be taken when interpreting long-term toxicity. In light of these data and published results, a long-term follow-up ( $>2$ years) and routine toxicity monitoring after SBRT are necessary.

Our study had some limitations that need to be discussed.
First, the results are based on retrospective data and the conditions of the patients were more complicated compared with prospective studies, which unavoidably enhanced the heterogeneity of our sample. Toxicity scoring in a retrospective study is also extremely challenging. Due to the limited data available on hospitalization at outside institutions, as well as the inclusion of patients at high risk or in poor health generally, these toxicities might not have been caused by radiation therapy. Secondly, the case samples were small and the results need to be demonstrated by a large sample prospective study. Thirdly, the enrolled patients were older, and advanced age can contribute to a poor prognosis. In the present study, the patients had a median age of 75 years, and $74.2 \%$ of them had a Charlson comorbidity index score $\geq 5$. This may explain $3(37.5 \%)$ of the 8 deaths from other diseases in current study. Besides, using the RECIST criteria to assess LC in this research carries obvious limitations in the post-radiotherapy setting (21).

In conclusion, for SBRT, central early-stage NSCLC tumors are not a "no fly zone". The current study validated that SBRT with a BED10 100-119 Gy in 4-10 fractions is effective and tolerable for patients central early-stage NSCLC. Further verification of our results in large-scale studies is necessary.

\section{Acknowledgments}

Funding: This study was supported by the Start-up Fund for Talent Introduction of Shanghai Pulmonary Hospital (Yaping Xu) (grant number: 20180101) and the Medical and Health Technology Project of Zhejiang Province (grant number: 201344326).

\section{Footnote}

Reporting Checklist: The authors have completed the STROBE reporting checklist. Available at http://dx.doi. org/10.21037/tlcr-20-851

Data Sharing Statement: Available at http://dx.doi. org/10.21037/tlcr-20-851

Conflicts of Interest: All authors have completed the ICMJE uniform disclosure form (available at http://dx.doi. org/10.21037/tlcr-20-851). The authors have no conflicts of interest to declare.

Ethical Statement: The authors are accountable for all 
aspects of the work in ensuring that questions related to the accuracy or integrity of any part of the work are appropriately investigated and resolved. The study protocol was approved by the human research ethics committee of our institution (approval ID: IRB-2020-140 $(\mathrm{Ke})$ ), and signed informed consent was obtained from all patients. The study was conducted in accordance with the Declaration of Helsinki (as revised in 2013).

Open Access Statement: This is an Open Access article distributed in accordance with the Creative Commons Attribution-NonCommercial-NoDerivs 4.0 International License (CC BY-NC-ND 4.0), which permits the noncommercial replication and distribution of the article with the strict proviso that no changes or edits are made and the original work is properly cited (including links to both the formal publication through the relevant DOI and the license). See: https://creativecommons.org/licenses/by-nc-nd/4.0/.

\section{References}

1. Bremnes RM, Busund LT, Kilvaer TL, et al. The Role of Tumor-Infiltrating Lymphocytes in Development, Progression, and Prognosis of Non-Small Cell Lung Cancer. J Thorac Oncol 2016;11:789-800.

2. Ball D, Mai GT, Vinod S, et al. Stereotactic ablative radiotherapy versus standard radiotherapy in stage 1 nonsmall-cell lung cancer (TROG 09.02 CHISEL): a phase 3 , open-label, randomised controlled trial. Lancet Oncol 2019;20:494-503.

3. Onishi H, Shirato H, Nagata Y, et al. Hypofractionated stereotactic radiotherapy (HypoFXSRT) for stage I nonsmall cell lung cancer: updated results of 257 patients in a Japanese multi-institutional study. J Thorac Oncol 2007;2:S94-S100.

4. Timmerman R, McGarry R, Yiannoutsos C, et al. Excessive toxicity when treating central tumors in a phase II study of stereotactic body radiation therapy for medically inoperable early-stage lung cancer. J Clin Oncol 2006;24:4833-9.

5. Xiao Y, Papiez L, Paulus R, et al. Dosimetric evaluation of heterogeneity corrections for RTOG 0236 : stereotactic body radiotherapy of inoperable stage I-II non-small-cell lung cancer. Int J Radiat Oncol Biol Phys 2009;73:1235-42.

6. Bezjak A, Paulus R, Gaspar LE, et al. Safety and Efficacy of a Five-Fraction Stereotactic Body Radiotherapy Schedule for Centrally Located Non-Small-Cell Lung Cancer: NRG Oncology/RTOG 0813 Trial. J Clin Oncol
2019;37:1316-25.

7. Duijm M, van der Voort van Zyp NC, van de Vaart P, et al. Predicting High-Grade Esophagus Toxicity After Treating Central Lung Tumors With Stereotactic Radiation Therapy Using a Normal Tissue Complication Probability Model. Int J Radiat Oncol Biol Phys 2020;106:73-81.

8. Arnett ALH, Mou B, Owen D, et al. Long-term Clinical Outcomes and Safety Profile of SBRT for Centrally Located NSCLC. Adv Radiat Oncol 2019;4:422-8.

9. Rulach R, McLoone P, Lumsden G, et al. Toxicity and Efficacy of Stereotactic Ablative Body Radiotherapy for Moderately Central Non-small Cell Lung Cancers Using 50 Gy in Five Fractions. Clin Oncol (R Coll Radiol) 2020;32:250-8.

10. Ma SJ, Syed YA, Rivers CI, et al. Comparison of singleand five-fraction schedules of stereotactic body radiation therapy for central lung tumours: a single institution experience. J Radiother Pract 2017;16:148-54.

11. Schanne DH, Nestle U, Allgauer $M$, et al. Stereotactic body radiotherapy for centrally located stage I NSCLC: a multicenter analysis. Strahlenther Onkol 2015;191:125-32.

12. Timmerman R, Heinzerling J, Abdulrahman R, et al. Stereotactic body radiation therapy for thoracic cancers: recommendations for patient selection, setup and therapy. Front Radiat Ther Oncol 2011;43:395-411.

13. Ricardi U, Frezza G, Filippi AR, et al. Stereotactic Ablative Radiotherapy for stage I histologically proven non-small cell lung cancer: an Italian multicenter observational study. Lung Cancer 2014;84:248-53.

14. Sun B, Brooks ED, Komaki RU, et al. 7-year follow-up after stereotactic ablative radiotherapy for patients with stage I non-small cell lung cancer: Results of a phase 2 clinical trial. Cancer. 2017;123:3031-9.

15. Chen Y, Guo W, Lu Y, Zou B. Dose-individualized stereotactic body radiotherapy for T1-3N0 non-small cell lung cancer: long-term results and efficacy of adjuvant chemotherapy. Radiother Oncol 2008;88:351-8.

16. Bernstein MB, Krishnan S, Hodge JW, et al. Immunotherapy and stereotactic ablative radiotherapy (ISABR): a curative approach? Nat Rev Clin Oncol 2016;13:516-24.

17. Golden EB, Demaria S, Schiff PB, et al. An abscopal response to radiation and ipilimumab in a patient with metastatic non-small cell lung cancer. Cancer Immunol Res. 2013;1:365-72.

18. Filatenkov A, Baker J, Mueller AM, et al. Ablative Tumor Radiation Can Change the Tumor Immune Cell Microenvironment to Induce Durable Complete 
Remissions. Clin Cancer Res 2015;21:3727-39.

19. ClinicalTrials.gov. Durvalumab vs Placebo Following Stereotactic Body Radiation Therapy in Early Stage Unresected Non-small Cell Lung Cancer Patients (PACIFIC-4). ClinicalTrialsgov Identifier: NCT03833154. Available online: https://clinicaltrials.gov/ct2/show/NCT0 3833154? id=NCT03833154\&draw=2\&rank=1\&load=cart 20. Lindberg K, Bergström P, Brustugun O, et al. OA24.05
The Nordic HILUS-Trial - First Report of a Phase II Trial of SBRT of Centrally Located Lung Tumors. J Thorac Oncol 2017;12:S340.

21. Mattonen SA, Ward AD, Palma DA. Pulmonary imaging after stereotactic radiotherapy-does RECIST still apply? Br J Radiol 2016;89:20160113.

(English Language Editor: J. Reynolds)

Cite this article as: Sun X, Li Y, Zhu Y, Li Q, Yuan X, Lin Q, Weng D, Xu Q, Liu H, Xu Y. Four-year follow-up outcomes after stereotactic body radiation therapy for central earlystage non-small cell lung cancer. Transl Lung Cancer Res 2020;9(4):1472-1482. doi: 10.21037/tlcr-20-851 


\section{Supplementary}
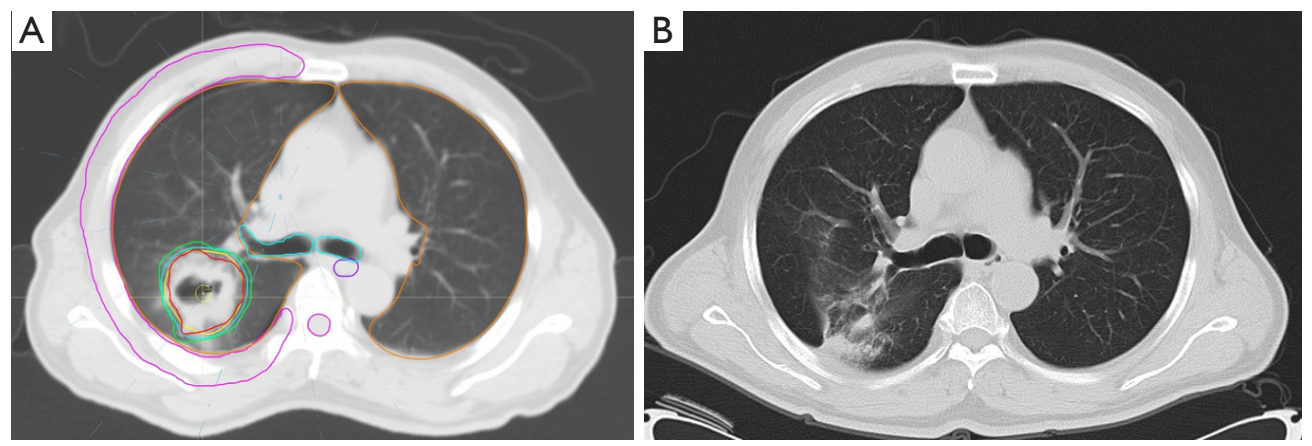

Figure S1 A representation of stereotactic body radiation therapy (SBRT) for central tumors. A 62-year-old man with stage IIA non-smallcell lung cancer. (A) Axial computed tomography image of pre-SBRT. The primary tumor was $5.0 \mathrm{~cm}$ in the greatest dimension as measured on a simulation computed tomography scan. The patient was treated with SBRT with 10 Gy $\times 5$ fractions. (B) 7 months after SBRT. The tumor was $1.4 \mathrm{~cm}$ in the greatest dimension as measured on a computed tomography scan. 